\title{
P I 0-08. Intracellular detection of differential APOBEC3G, TRIM5a, and LEDGF/p75 expression in peripheral blood from HIV-I infected subjects by flow cytometry
}

\author{
K Mous*1, W Jennes ${ }^{2}$, L Kestens ${ }^{2}$ and X Van Ostade ${ }^{1}$
}

Address: ${ }^{1}$ University of Antwerp, Wilrijk, Belgium and ${ }^{2}$ Institute of Tropical Medicine, Antwerp, Belgium

* Corresponding author

from AIDS Vaccine 2009

Paris, France. 19-22 October 2009

Published: 22 October 2009

Retrovirology 2009, 6(Suppl 3):PI39 doi:I0.II86/I742-4690-6-S3-PI39

This abstract is available from: http://www.retrovirology.com/content/6/S3/PI39

(C) 2009 Mous et al; licensee BioMed Central Ltd.

\section{Background}

HIV-1 replication in host cells depends on a delicate balance between intrinsic immune factors limiting viral dissemination like APOBEC3G and TRIM5alpha, and cellular co-factors required for virus propagation like LEDGF/p75. Thus far, host protein expression levels involved in HIV-1 replication have been determined mainly through mRNA-based real-time PCR and proteinbased western blotting. We analyzed protein expression at single-cell level using flow cytometry.

\section{Methods}

Following adaptation of established intracellular cytokine staining protocols, blocking and stimulation experiments were conducted to address the method's specificity. Further optimization and reproducibility testing were performed prior to application of the method. Ten HIV-1 negative, 10 therapy-naive HIV-1 positive, and 10 HAARTtreated HIV-1 positive persons were enrolled. Subsequently, differential APOBEC3G, TRIM5alpha, and LEDGF/p75 expression in peripheral blood mononuclear cell subsets were analyzed.

\section{Results}

The method was shown to be specific and reproducible. CD4+ T cells showed significantly lower expression of APOBEC3G and TRIM5alpha and significantly higher expression of LEDGF/p75 than monocytes, which is in line with the differential tropism of HIV-1 for these cell subsets. Similarly, HIV-1 susceptible CD16+ monocytes showed higher expression levels of LEDGF/p75 than CD16- monocytes, albeit without differences in APOBEC3G or TRIM5alpha expression. Expression of APOBEC3G and TRIM5alpha, but not LEDGF/p75, tended to be lower among therapy-naive HIV-1 patients than among HIV-1 negative controls. The differential expression of A3G and TRIM5alpha relative to LEDGF/ p75 was further supported by the presence of direct correlations between A3G and TRIM5alpha, whilst weak or no correlations were observed with LEDGF/p75.

\section{Conclusion}

These data show that APOBEC3G, TRIM5alpha, and LEDGF/p75 are differentially expressed in mononuclear cell subsets of HIV-1 patients and controls in a way that is consistent with the known functions of these factors during HIV-1 replication. Analysis of constitutively expressed host factors at single-cell level may prove useful for our further understanding of virus-host interactions. 